\title{
Scattering of Low-Energy Photons by Particles of Spin $\frac{1}{2}^{*} \dagger \ddagger$
}

\author{
M. Gell-Mann and M. L. Goldberger $\S$ \\ Institute for Nuclear Studies and Department of Physics, University of Chicago, Chicago, Illinois
}

(Received September 3, 1954)

\begin{abstract}
The first two terms in a frequency expansion of the photon scattering amplitude are considered in the case where the scatterer is a particle of spin $\frac{1}{2}$. It is shown that an exact calculation in quantum field theory gives results identical with those obtained by classical methods or else by use of the Dirac equation with an anomalous Pauli moment. The results depend only on the charge, mass, and magnetic moment of the scatterer. In the case of the proton, the second term, arising from scattering by the magnetic moment, appears to be negligible in comparison with effects due to Thomson and Rayleigh scattering.
\end{abstract}

\section{INTRODUCTION}

$I^{\top}$ Thas long been known that the scattering of photons in the limit of zero frequency is described correctly by the classical Thomson amplitude, which depends, of course, only on the charge and mass of the scattering particle. The result is surely independent of the spin of the particle. In the case where the spin is $\frac{1}{2}$, however, explicit proofs have been given that the result of the classical calculation is reproduced in quantum mechanics: for a normal Dirac particle, in the lowest order of perturbation theory, the proof is contained in the famous calculation of Klein and Nishina; ${ }^{1}$ it has since been shown by Thirring ${ }^{2}$ and by Kroll and Ruderman ${ }^{3}$ that the Thomson formula is exactly correct in any local, renormalizable field theory, including, for example, electromagnetic and mesonic radiative corrections.

In general, the amplitude for the scattering of photons by a particle of spin $\frac{1}{2}$ may be expanded in a power series in the frequency of the photon. The Thomson amplitude is the zeroth term in such an expansion. It is the next term, linear in frequency, that we shall consider here. We shall calculate the linear term in three different ways: (1) Classically, using Kramer's classical description $^{4}$ of a Dirac particle; besides the Dirac magnetic moment considered by Kramers, a classical anomalous moment is added. (2) In quantum mechanics, for a Dirac particle with a Pauli anomalous magnetic moment, in the lowest order of perturbation theory. (3) Exactly in quantum field theory, for a Dirac particle interacting with arbitrary local and renormalizable fields, for example photons and mesons.

\footnotetext{
* Some of the work presented here was reported at the Glasgow International Nuclear Physics Conference, 1954, and is summarized in the Conference Proceedings.

$\dagger$ Supported by grants from the U. S. Atomic Energy Commission.

$\ddagger$ The authors are indebted to Dr. Francis E. Low for sending them a copy of his paper on the same subject before publication His results and ours are in complete agreement although obtained by different methods.

$\S$ Visiting Scientist at Brookhaven National Laboratory while part of this work was carried out.

1 O. Klein and Y. Nishina, Z. Physik 52, 853, 869 (1929).

2 W. Thirring, Phil. Mag. 41, 1193 (1950).

${ }^{3}$ N. Kroll and M. Ruderman, Phys. Rev. 93, 233 (1954)

${ }^{4}$ H. A. Kramers, Grundlagen der Quantentheorie (Edwards Brothers, Ann Arbor, 1944), p. 227 ff.
}

The results of the three calculations are identical and show that the linear term depends only on the charge, mass, and static magnetic moment of the scattering particle.

\section{CLASSICAL CALCULATION}

Let us consider an incoming plane light wave with the electric field $\mathbf{E}_{0}$ given by ${ }^{\mathbf{5}}$

$$
\mathbf{E}_{0}=\mathbf{e} \exp (i \mathbf{q} \cdot \mathbf{r}-i q t) \text {. }
$$

The scatterer is taken to be at rest at the origin initially $(t=0)$. After a long time and at great distance, a scattered wave appears with the asymptotic electric field given (to first order in the incoming field strength) by

$$
\mathbf{E}_{s c}=\mathbf{G}\left(\mathbf{n}^{\prime}, \mathbf{n}\right) \cdot \mathbf{e} r^{-1} \exp [i q(r-t)]
$$

where $\mathbf{G}\left(\mathbf{n}^{\prime}, \mathbf{n}\right)$ is a dyadic describing the scattering from the incident direction along the unit vector $\mathbf{n} \equiv \mathbf{q} / q$ to the final direction along the unit vector $\mathbf{n}^{\prime} \equiv \mathbf{q}^{\prime} / q^{\prime}$. The scattering amplitude ${ }^{6} f$ is now defined by the equation,

$$
f=\mathbf{e}^{\prime} \cdot \mathbf{G}\left(\mathbf{n}^{\prime}, \mathbf{n}\right) \cdot \mathbf{e},
$$

in terms of a final polarization vector $\mathbf{e}^{\prime}$ orthogonal to $\mathbf{n}^{\prime}$.

The scattering particle, with charge $e$ and mass $M$, is characterized by its position vector $\mathbf{R}(t)$ and a spin angular momentum $\mathbf{S}(t)$ that is constant in magnitude but free to rotate. The magnetic moment $\boldsymbol{u}(t)$ is taken to be proportional to $\mathbf{S}(t)$; the gyromagnetic ratio $g$ will be written as the sum of the "normal" Dirac value $e / M$ and an "anomalous" part $g_{A}$. Of course the Dirac $g$-factor is often thought to be anomalous from the classical point of view. However Kramers has presented a

${ }^{5}$ We have set $c=1$; we will further put $\hbar=1$, and use units in which $e^{2} / \hbar c=1 / 137$

${ }^{6}$ Strictly speaking, there is a frequency dependent correction factor by which Eq. (2.3) must be multiplied in order that it be the exact scattering amplitude in the laboratory system. This factor arises classically from the Doppler shift of the radiation emitted by the particle which is set into motion by the incident wave. In quantum theory the factor is precisely $q^{\prime} / q$ where the frequency ratio is computed from the Compton law. We shall continue to employ the same quantity, $f$, in quantum theory, where it is equal to the correct scattering amplitude in the centerof-mass system, and also to the Feynman matrix element in either system. 
classical theory ${ }^{4}$ in which the Dirac $g$-factor appears naturally. In describing the interaction of the scattering particle with the electromagnetic field, we shall make use of Kramers' formulation of the problem.

Kramer's equations of motion for a classical Dirac particle may be derived from the following Lagrangian (accurate to order $V / c$ ):

$$
\begin{aligned}
L_{K}=\frac{1}{2} M \mathbf{V}^{2}+\frac{1}{8} \pi \int\left(\mathbf{E}^{2}-\mathbf{H}^{2}\right) d^{3} r+e \mathbf{V} \cdot \mathbf{A}(\mathbf{R}) \\
+\frac{e}{M} \mathbf{S} \cdot\left[\mathbf{H}(\mathbf{R})-\frac{1}{2} \mathbf{V} \times \mathbf{E}(\mathbf{R})\right],
\end{aligned}
$$

where $\mathbf{V}=\partial \mathbf{R} / \partial t$, $\mathbf{A}$ is the transverse vector potential, and $\mathbf{E}$ and $\mathbf{H}$ are given, of course, by the equations

$$
\mathbf{E}=-\partial \mathbf{A} / \partial t, \quad \mathbf{H}=\boldsymbol{\nabla} \times \mathbf{A} .
$$

In Eq. (2.4), the scalar potential has been ignored since it does not contribute to the scattering of light waves. The factor of $\frac{1}{2}$ in the last term is the celebrated Thomas factor.

In order to include the effects of the anomalous magnetic moment, we add to the Lagrangian of Kramers the additional quantity

$$
L_{A}=g_{A} \mathbf{S} \cdot[\mathbf{H}(\mathbf{R})-\mathbf{V} \times \mathbf{E}(\mathbf{R})] .
$$

The absence of the Thomas factor in the case of the anomalous moment is familiar. From the total Lagrangian $L_{K}+L_{A}$ we obtain the following equations of motion for field and particle:

$$
\begin{gathered}
\boldsymbol{\nabla} \times \mathbf{E}+\partial \mathbf{H} / \partial t=0 \\
\boldsymbol{\nabla} \times \mathbf{H}-\partial \mathbf{E} / \partial t=4 \pi \mathbf{J}=4 \pi e \mathbf{V} \delta(\mathbf{r}-\mathbf{R}) \\
+4 \pi \boldsymbol{\nabla} \times[g \mathbf{S} \delta(\mathbf{r}-\mathbf{R})] \\
+4 \pi \frac{d}{d t}\left[\left(\frac{e}{2 M}+g_{A}\right) \mathbf{V} \times \mathbf{S} \delta(\mathbf{r}-\mathbf{R})\right], \\
M \partial^{2} \mathbf{R} / \partial t^{2}=e \mathbf{E}(\mathbf{R})+g_{A}(\mathbf{S} \cdot \boldsymbol{\nabla}) \mathbf{H} \\
+\frac{e}{M} \frac{(\mathbf{S} \cdot \boldsymbol{\nabla}) \mathbf{H}+\boldsymbol{\nabla}(\mathbf{S} \cdot \mathbf{H})}{2}, \\
\partial \mathbf{S} / \partial t=g \mathbf{S} \times \mathbf{H} .
\end{gathered}
$$

In Eqs. (2.7) and (2.8) we have simply two of Maxwell's equations. The current in Eq. (2.8) comprises three terms : the ordinary translational current; the curl of the density of magnetization; and a displacement current which arises from the fact that a moving magnetized medium acquires an effective electric polarization. Only the last term is affected by the Thomas factor. In Eq. (2.9), the forces acting on the particle are exhibited. We have omitted the self-force and also other terms, such as the Lorentz force, which depend on the field-induced velocity and angular acceleration of the particle and are therefore effectively quadratic in the field strengths. We have left only the electric force and the interaction of the magnetic moment with the gradient of the magnetic field. The Dirac moment, affected by the Thomas factor, behaves as if half of it were due to a genuine magnetic dipole and half to a current loop; the anomalous moment behaves entirely as a point dipole. Equation (2.10) is merely the usual formula for the torque on a magnetic dipole; again terms effectively quadratic in the field strengths have been dropped.

The asymptotic electric field may now be computed from the relation.

$$
\begin{aligned}
& \mathbf{E} \rightarrow \mathbf{E}_{0}(\mathbf{r}, t)+\mathbf{E}_{s c}(\mathbf{r}, t) \\
&= \mathbf{E}_{0}-r^{-1} \int d^{3} r^{\prime} \int_{-\infty}^{\infty} d t^{\prime}(\partial / \partial t) \mathbf{J}_{\perp}\left(\mathbf{r}^{\prime}, t^{\prime}\right) \\
& \times \delta\left(t^{\prime}-t+r-r^{-1} \mathbf{r} \cdot \mathbf{r}^{\prime}\right),
\end{aligned}
$$

in which $\mathbf{J}_{\perp}$ is the part of the current that is perpendicular to $\mathbf{r}$, i.e., to the direction of observation. In substituting into Eq. (2.11) the formula (2.8) for the current, we may simplify the result by omitting higher order terms in the field strengths. (It must be borne in mind that $\mathbf{R}, \partial \mathbf{R} / \partial t, \partial^{2} \mathbf{R} / \partial t^{2}, \partial \mathbf{S} / \partial t$, and $\partial^{2} \mathbf{S} / \partial t^{2}$ would all be zero in the absence of the incident field.) We find

where

$$
\mathbf{E}_{s c}(\mathbf{r}, t)=-r^{-1} \mathbf{Z}_{\perp}(t-r),
$$

$$
\begin{array}{r}
\mathbf{Z}=e \frac{\partial^{2} \mathbf{R}}{\partial t^{2}}-g r^{-1}\left(\mathbf{r} \times \frac{\partial^{2} \mathbf{S}}{\partial t^{2}}\right)-g r^{-2}\left(\mathbf{r} \cdot \frac{\partial^{3} \mathbf{R}}{\partial t^{3}}\right)(\mathbf{r} \times \mathbf{S}) \\
-\left(\frac{e}{2 M}+g_{A}\right)\left(\mathbf{S} \times \frac{\partial^{3} \mathbf{R}}{\partial t^{3}}\right) .
\end{array}
$$

Finally, we must substitute into Eq. (2.13) the equations of motion of Eqs. (2.9) and (2.10), simplifying as before. We obtain, up to terms linear in the frequency, the expression

$$
\begin{aligned}
& \mathbf{Z}=\left\{\frac{e^{2}}{M} \mathbf{e}+i g^{2} q \mathbf{n}^{\prime} \times[\mathbf{S} \times(\mathbf{n} \times \mathbf{e})]\right. \\
& +\frac{i e g}{M} q\left[(\mathbf{n} \cdot \mathbf{S})(\mathbf{n} \times \mathbf{e})+\left(\mathbf{n}^{\prime} \cdot \mathbf{e}\right)\left(\mathbf{n}^{\prime} \times \mathbf{S}\right)\right] \\
& \left.+\frac{i e}{M}\left(\frac{e}{2 M}+g_{A}\right) q(\mathbf{S} \times \mathbf{e})\right\} \exp [i q(r-t)],
\end{aligned}
$$

so that the scattering amplitude defined by Eq. (2.3) is given by the equation

$$
\begin{array}{r}
f=-\frac{e^{2}}{M}\left(\mathbf{e}^{\prime} \cdot \mathbf{e}\right)-i g^{2} q \mathbf{S} \cdot\left[\left(\mathbf{n}^{\prime} \times \mathbf{e}^{\prime}\right) \times(\mathbf{n} \times \mathbf{e})\right] \\
-\frac{i e g}{M} q\left[\mathbf{S} \cdot\left\{\frac{\mathbf{n}(\mathbf{n} \times \mathbf{e})+(\mathbf{n} \times \mathbf{e}) \mathbf{n}}{2}\right\} \cdot \mathbf{e}^{\prime}\right. \\
\left.-\mathbf{S} \cdot\left\{\frac{\mathbf{n}^{\prime}\left(\mathbf{n}^{\prime} \times \mathbf{e}^{\prime}\right)+\left(\mathbf{n}^{\prime} \times \mathbf{e}^{\prime}\right) \mathbf{n}^{\prime}}{2}\right\} \cdot \mathbf{e}^{\prime}\right] \\
+\frac{i e g_{A}}{M} q \mathbf{S} \cdot\left(\mathbf{e}^{\prime} \times \mathbf{e}\right) .
\end{array}
$$


Here $\mathbf{S}$ is the initial value of the spin angular momentum.

It is not difficult to trace through Eqs. (2.13)-(2.15) the meaning of the various terms in the scattering amplitude. The first term is, of course, the Thomson amplitude. The second represents magnetic dipole scattering; the magnetic moment absorbs $M 1$ radiation, precesses, and re-emits $M 1$ radiation. The first half of the third term represents the absorption of $M 2$ radiation (through the interaction of the magnetic moment of the particle with the gradient of the magnetic field of the incident wave) followed by the emission of $E 1$ radiation because of the resulting acceleration. The second half of the third term describes the reverse process; the particle absorbs $E 1$ radiation and is accelerated, emitting $M 2$ radiation by virtue of the magnetic moment coupling. Now the translational force on the particle due to the anomalous part of the magnetic moment is not of pure $M 2$ origin, as can be seen from Eq. (2.9); there is an $E 1$ part as well. Hence the fourth term in the scattering amplitude, representing $E 1$ scattering.

\section{QUANTUM-MECHANICAL CALCULATIONS}

The scattering of photons by a Dirac particle with a Pauli anomalous magnetic moment, without radiative corrections, has been discussed by Powell. ${ }^{7}$ The wave function of the scatterer obeys the equation

$$
\left\{\gamma_{\mu}\left(\partial / \partial x_{\mu}-i e A_{\mu}\right)+M-\frac{1}{2} \mu_{A} \sigma_{\mu \nu} F_{\mu \nu}\right\} \psi=0 .
$$

The quantum-mechanical scattering amplitude is most easily calculated as the sum of the two Feynman matrix elements corresponding to absorption followed by emission and vice versa. The result is, up to terms linear in the frequency,

$$
\begin{aligned}
& f=- \frac{e^{2}}{M}\left(\mathbf{e}^{\prime} \cdot \mathbf{e}\right)-2 i \mu^{2} q \mathbf{\sigma} \cdot\left[\left(\mathbf{n}^{\prime} \times \mathbf{e}^{\prime}\right) \times(\mathbf{n} \times \mathbf{e})\right] \\
&-\frac{i e \mu}{M} q\left[\boldsymbol{\sigma} \cdot\left\{\frac{\mathbf{n}(\mathbf{n} \times \mathbf{e})+(\mathbf{n} \times \mathbf{e}) \mathbf{n}}{2}\right\} \cdot \mathbf{e}^{\prime}\right. \\
&\left.-\boldsymbol{\sigma} \cdot\left\{\frac{\mathbf{n}^{\prime}\left(\mathbf{n}^{\prime} \times \mathbf{e}^{\prime}\right)+\left(\mathbf{n}^{\prime} \times \mathbf{e}^{\prime}\right) \mathbf{n}^{\prime}}{2}\right\} \cdot \mathbf{e}\right] \\
&+\frac{i e \mu_{A}}{M} q \boldsymbol{\sigma} \cdot\left(\mathbf{e}^{\prime} \times \mathbf{e}\right) .
\end{aligned}
$$

The correspondence with the classical formula (2.15) is perfect; we must of course identify $\mathbf{S}$ with $\boldsymbol{\sigma} / 2, g$ with $2 \mu$, and $g_{A}$ with $2 \mu_{A}$.

We must now demonstrate that Eq. (3.2) is rigorously correct in quantum field theory (except, of course, for higher terms in the frequency). We abandon the phenomenological Pauli moment; instead we suppose that the scatterer is a normal Dirac particle, coupled to

\footnotetext{
${ }^{7}$ J. L. Powell, Phys. Rev. 75, 32 (1949).
}

fields describing photons, mesons, etc. The anomalous moment is attributed to the effects of these couplings. In what follows, the interaction with photons must have conventional local, gauge-invariant character and all interactions must be renormalizable; otherwise we need not specify the nature of the fields and interactions in detail. We shall employ the language of Feynman diagrams, but it should be emphasized that no use is made of perturbation theory.

Let us refer to the scatterer as a nucleon, for definiteness. We may picture the entire sequence of Feynman diagrams in the following way: The nucleon line proceeds from the beginning to the end of the diagram, emitting and absorbing, in between, various virtual quanta that belong to its self-field. The initial and final real photon lines terminate on charge-bearing lines, which may correspond to the nucleon itself or to virtual mesons or members of pairs, etc. We may divide all the diagrams into two classes, $A$ and $B .{ }^{8}$ In Class $A$ we include all the diagrams in which those parts that include the terminations of the two real photon lines are separated by a strength of bare nucleon line, i.e., nucleon line free of virtual quanta. Class $B$ comprises all other diagrams, those in which there is no stretch of bare nucleon line between the absorption and the emission of the real photons.

For the sum of all diagrams of Class $A$, we can write a simple closed expression. Let $q$ and $q^{\prime}$ be the initial and final four-momenta of the photon, ${ }^{9}$ and $p=(0,0,0, i M)$ be the initial four-momentum of the nucleon. The indices $\mu$ and $\nu$ will denote the initial and final direction of polarization. Then the contribution of diagrams of class $A$ to the scattering amplitude is

where

$$
T_{\nu \mu}^{(A)}\left(q^{\prime}, q\right)=t_{\nu \mu}\left(q^{\prime}, q\right)+t_{\mu \nu}\left(-q,-q^{\prime}\right)
$$

$$
\begin{aligned}
t_{p \mu}\left(q^{\prime}, q\right)=-e^{2} \bar{\psi}_{f}\left(p+q-q^{\prime}\right) \Gamma_{\nu}\left(p+q-q^{\prime}, p+q\right) \\
\times S(p+q) \Gamma_{\mu}(p+q, p) \psi_{i}(p) .
\end{aligned}
$$

Here $\psi_{i}(p)$ is the initial Dirac spinor; $\Gamma_{\mu}(p+q, p)$ is the exact renormalized vertex operator corresponding to the absorption of the initial photon; $S(p+q)$ is the exact renormalized propagation function for the virtual state of the nucleon with momentum $p+q ; \Gamma_{\nu}(p+q$ $\left.-q^{\prime}, p+q\right)$ is the vertex operator for the emission of the final photon; and $\bar{\psi}_{f}\left(p+q-q^{\prime}\right)$ is the final Dirac spinor. The second term in Eq. (3.3) describes the "crossed diagrams" in which emission of the final photon precedes absorption of the initial one.

It is evident from Eq. (3.3) that the partial scattering amplitude $T^{(A)}$ obeys a symmetry relation; this relation actually holds for the total scattering amplitude $T$ and

${ }^{8}$ For a discussion of a similar calculation, see Appendix of paper by Deser, Thirring, and Goldberger, Phys. Rev. 94, 711 (1954).

${ }^{9}$ In the remainder of this section, $q$ stands for the four-vector momentum of the incident photon. The photon frequency, which is denoted elsewhere by $q$, is here called $q_{0}$. 
is given by the equation

$$
T_{\nu \mu}\left(q^{\prime}, q\right)=T_{\mu \nu}\left(-q,-q^{\prime}\right)
$$

This symmetry condition depends only on the fact that for every "crossed" diagram there is a corresponding "uncrossed" one and vice versa.

To proceed further with the evaluation of $T^{(A)}$ up to terms linear in frequency, we must exhibit expansions of $\Gamma, S$, and $\psi_{f}$ in powers of $q$ and $q^{\prime}$. We write the propagation function in the form

$S(p+q)=\left\{i \gamma \cdot(p+q) G\left[(p+q)^{2}\right]+M F\left[(p+q)^{2}\right]\right\}^{-1}$,

and, expanding, we find ${ }^{10}$

$$
\begin{aligned}
S(p+q) \simeq & \frac{1}{2 p \cdot q F}[(-i \gamma \cdot p+M) F-i \gamma \cdot q F \\
& +2 p \cdot q\left(-i \gamma \cdot p G^{\prime}+M F^{\prime}\right)-i \gamma \cdot q(2 p \cdot q) G^{\prime} \\
& +2(p \cdot q)^{2}\left(-i \gamma \cdot p G^{\prime \prime}+M F^{\prime \prime}\right) \\
& -2 p \cdot q\left\{2 G^{\prime}+M^{2}\left(F^{\prime \prime}-G^{\prime \prime}+\frac{F^{2}-G^{2}}{F}\right)\right\} \\
& \left.\times\left\{2 p \cdot q\left(-i \gamma \cdot p G^{\prime}+M F^{\prime}\right)-F i \gamma \cdot q\right\}\right] .
\end{aligned}
$$

The functions $F, F^{\prime}$, etc. are evaluated at $p^{2}=-M^{2}$. It should be noted that not all terms linear in $q$ have been retained, but only those that contribute to (3.4) in the required order. The form of the vertex operator, like that of the propagation function, can be specified on general grounds of invariance; one finds, to first order in the frequency,

$$
\begin{aligned}
& \Gamma_{\mu}(p+q, q) \\
& =\Gamma_{\mu}(p, p)+\frac{1}{2} q_{\lambda} \frac{\partial}{\partial p_{\lambda}} \Gamma_{\mu}(p, p)+\frac{p \cdot q}{M^{3}} \sigma_{\mu \lambda} p_{\lambda} F_{1}\left(p^{2}\right) \\
& \quad+\frac{1}{2 M} \sigma_{\mu \lambda} q_{\lambda} F_{2}\left(p^{2}\right)+\frac{q_{\lambda} \sigma_{\lambda \rho} p_{\rho} p_{\mu}}{M^{3}} F_{3}\left(p^{2}\right) ; \\
& \Gamma_{\nu}\left(p+q-q^{\prime}, p+q\right) \\
& =\Gamma_{\nu}(p, p)+\frac{1}{2}\left(2 q_{\lambda}-q_{\lambda}{ }^{\prime}\right) \frac{\partial}{\partial p_{\lambda}} \Gamma_{\nu}(p, p) \\
& \quad-\frac{p \cdot q^{\prime}}{M^{3}} \sigma_{\nu \lambda} p_{\lambda} F_{1}\left(p^{2}\right)-\frac{1}{2 M} \sigma_{\nu \lambda} q_{\lambda}^{\prime} F_{2}\left(p^{2}\right) \\
& -\frac{q_{\lambda}^{\prime} \sigma_{\lambda \rho} p_{\rho} p_{\nu}}{M^{3}} F_{3}\left(p^{2}\right) .
\end{aligned}
$$

Among the symmetry principles useful in deriving (3.8) and (3.9) are those related to charge conjugation

\footnotetext{
${ }^{10}$ Primes attached to $F$ and $G$ indicate differentiation with
} respect to $p^{2}$. and space-time inversion:

$$
\begin{gathered}
C^{-1} \Gamma_{\mu}\left(p_{1}, p_{2}\right) C=-\Gamma_{\mu}^{T}\left(-p_{2},-p_{1}\right), \\
\gamma_{5} \Gamma_{\mu}\left(p_{1}, p_{2}\right) \gamma_{5}=-\Gamma_{\mu}\left(-p_{1},-p_{2}\right),
\end{gathered}
$$

where $C$ is the charge-conjugation matrix. The vertex operator for equal arguments is connected with the propagation function through Ward's identity:

$$
\begin{aligned}
\Gamma_{\mu}(p, p)=\frac{1}{i} \frac{\partial}{\partial p_{\mu}} S^{-1}(p)=\gamma_{\mu} G\left(p^{2}\right) \\
-2 i p_{\mu}\left\{i \gamma \cdot p G^{\prime}\left(p^{2}\right)+M F^{\prime}\left(p^{2}\right)\right\} .
\end{aligned}
$$

Furthermore, we may make use of the fact that for small values of $i \gamma \cdot p+M$, the reciprocal of the propagation function has the form

$$
S^{-1}(p)=i \gamma \cdot p+M+\sigma(i \gamma \cdot p+M)^{2}
$$

we then obtain the identities

$$
\begin{gathered}
F=G, \\
F+2 M^{2}\left(F^{\prime}-G^{\prime}\right)=1,
\end{gathered}
$$

where the quantities are evaluated at $p^{2}=-M^{2}$. Finally we must expand the spinors $\psi_{i}(p)$ and $\bar{\psi}_{f}\left(p+q-q^{\prime}\right)$ in powers of the frequency $:^{11}$

$$
\begin{gathered}
\psi_{i}(p)=\left(\begin{array}{l}
u \\
0
\end{array}\right), \\
\bar{\psi}_{f}\left(p+q-\zeta^{\prime}\right)=\left(v,-\boldsymbol{\sigma} \cdot\left(\mathbf{q}-\mathbf{q}^{\prime}\right) v / 2 M\right) .
\end{gathered}
$$

The evaluation of $T^{(A)}$ is completely straightforward in terms of the quantities introduced above; we shall simply give the result. To facilitate comparison with previous formulae, we write $e_{\nu} T_{\nu \mu}{ }^{(A)} e_{\mu}=v^{*} f^{(A)} u$, with

$$
\begin{aligned}
f^{(A)}=-\frac{e^{2}}{M} \mathbf{e}^{\prime} \cdot \mathbf{e} F-2 i \frac{e^{2}}{4 M^{2}}\left(F-F_{2}\right)^{2} q_{0} \boldsymbol{\sigma} \cdot\left(\mathbf{n}^{\prime} \times \mathbf{e}^{\prime}\right) \times(\mathbf{n} \times \mathbf{e}) \\
-\frac{i e}{M} \frac{e}{2 M}\left(F-F_{2}\right) q_{0}\left[\mathbf{\sigma} \cdot\{\mathbf{n} \mathbf{n} \times \mathbf{e}+\mathbf{n} \times \mathbf{e n}\} \cdot \mathbf{e}^{\prime}\right. \\
\left.-\mathbf{\sigma} \cdot\left\{\mathbf{n}^{\prime} \mathbf{n}^{\prime} \times \mathbf{e}^{\prime}+\mathbf{n}^{\prime} \times \mathbf{e}^{\prime} \mathbf{n}^{\prime}\right\} \cdot \mathbf{e}\right] \\
+\frac{i e}{M} \frac{e}{2 M}\left(F-F_{2}-1+4 F_{1}\right) q_{0} \boldsymbol{\sigma} \cdot \mathbf{e}^{\prime} \times \mathbf{e} .
\end{aligned}
$$

We must still evaluate the sum of the contributions from all diagrams of class $B$. It may be expressed concisely as follows, to first order in the frequency:

$$
\begin{aligned}
T_{\nu \mu}{ }^{(B)}\left(q^{\prime}, q\right)= & -i e^{2} \bar{\psi}_{f}\left(p+q-q^{\prime}\right) \\
& \times\left\{\frac{\partial}{\partial p_{\nu}} \Gamma_{\mu}(p, p)+\frac{\partial}{\partial p_{\nu}} q_{\lambda} \frac{\partial}{\partial p_{\lambda}{ }^{\prime}} \Gamma_{\mu}\left(p^{\prime}, p\right)\right. \\
& \left.-\frac{\partial}{\partial p_{\mu}} q_{\lambda} \frac{\partial}{\partial p_{\lambda^{\prime}}{ }^{\prime}} \Gamma_{\nu}\left(p^{\prime}, p\right)\right\}_{p^{\prime}=p} \psi_{i}(p) .
\end{aligned}
$$

${ }^{11} u$ and $v$ are two-component Pauli spinors describing the initial and final states. 
Using the expansions and relations given above, we obtain from (3.15) the simple result

$$
f^{(B)}=-\frac{e^{2}}{M} \mathbf{e}^{\prime} \cdot \mathbf{e}(1-F)-\frac{i e}{M} \frac{e q_{0}}{2 M} 4 F_{1} \mathbf{\sigma} \cdot\left(\mathbf{e}^{\prime} \times \mathbf{e}\right) .
$$

Adding $f^{(B)}$ to $f^{(A)}$ as given in (3.14), we see that we have exactly the formula (3.2) for the total scattering amplitude, provided we can set

$$
\mu_{A}=(e / 2 M)\left(F-F_{2}-1\right) .
$$

In order to prove Eq. (3.17), we consider the scattering of a nucleon by an external electromagnetic potential that transfers four-momentum $q$ to the nucleon. The transition matrix element $R$ is

$$
R=-\bar{\psi}(p+q) i e \Gamma_{\mu}(p+q, p) A_{\mu}(q) \psi(p) .
$$

If we choose $A$ to correspond to a uniform magnetic field and $p$ to a nucleon at rest, we may compute the term in $R$ proportional to $q$, using (3.8) and (3.13):

$$
R \simeq-\bar{\psi}(p)(e / 2 M)\left(F-F_{2}\right) \frac{1}{2} \sigma_{\mu \nu} F_{\mu \nu} \psi(0) .
$$

We may also compute $R$ on the basis of Eq. (3.1) for a Dirac particle with a Pauli moment:

$$
R \simeq-\bar{\psi}(p)\left(e / 2 M+\mu_{A}\right) \frac{1}{2} \sigma_{\mu \nu} F_{\mu \nu} \psi(p) .
$$

We conclude that Eq. (3.17) is correct.

\section{DISCUSSION}

We have derived an expression for the scattering amplitude up to terms linear in the photon frequency, and the expression is valid for any known theory. It is in order to discuss what predictions, if any, can be made of quantities that are likely to be measured experimentally. Let us first see what information we have about cross sections on the basis of our formula for the scattering amplitude.

For a neutral particle, we simply put $e=0$ and $\mu=\mu_{A}$ in Eq. (3.2). If we square the amplitude and sum and average over spins and polarizations, we obtain the leading term in an expansion of the cross section in powers of the frequency:

$$
d \sigma / d \Omega \simeq 4 q^{2} \mu^{4}\left(1+\frac{1}{2} \sin ^{2} \vartheta\right)
$$

For a charged particle, the leading term is of course the Thomson cross section. The next term, quadratic in frequency, is composed of two contributions, only one of which we can calculate by squaring the linear term in the scattering amplitude (3.2). The other contribution is made by the interference of the Thomson amplitude and the quadratic term in the scattering amplitude, which should come largely from Rayleigh scattering and which we have not hitherto discussed. If we denote the second contribution by $q^{2} I(\vartheta)$, then we have

$$
\begin{aligned}
\frac{d \sigma}{d \Omega}= & \frac{e^{4}}{M^{2}} \frac{\left(1+\cos ^{2} \vartheta\right)}{2}+q^{2}\left[I(\vartheta)+\left\{6 \mu^{4}+\frac{e^{2} \mu^{2}}{M^{2}}\right.\right. \\
& \left.-\frac{e^{3}}{M^{3}} \mu+\frac{3}{8} \frac{e^{4}}{M^{4}}\right\}+\cos \vartheta\left\{-\frac{8 e \mu^{3}}{M}+\frac{2 e^{2} \mu^{2}}{M^{2}}\right\} \\
& \left.+\cos ^{2} \vartheta\left\{-2 \mu^{4}+\frac{3 e^{2} \mu^{2}}{M^{2}}-\frac{e^{3} \mu}{M^{3}}-\frac{e^{4}}{8 M^{4}}\right\}\right]+\cdots
\end{aligned}
$$

In general there is no way to compute $I(\vartheta)$ without a detailed study of the mechanism of Rayleigh scattering for the particular case in question; in the forward direction, however, there is a useful general formula ${ }^{12}$ for $I$ :

$$
I(0)=-\frac{e^{2}}{M \pi^{2}} \int_{0}^{\infty} \frac{\sigma_{T}\left(q^{\prime}\right)}{q^{\prime 2}},
$$

where $\sigma_{T}\left(q^{\prime}\right)$ is the total cross section in the laboratory system for light of frequency $q^{\prime}$ incident on the scatterer. Equation (3.23) may be deduced from the Thomson formula and the principle that a light signal cannot be transmitted with velocity greater than $c$. Using Eqs. (3.22) and (3.23), we have for the forward direction:

$$
\left(\frac{d \sigma}{d \Omega}\right)_{0^{\circ}}=\frac{e^{4}}{M^{2}}+q^{2}\left\{-\frac{e^{2}}{M \pi^{2}} \int_{0}^{\infty} \frac{\sigma_{T}\left(q^{\prime}\right)}{q^{\prime 2}} d q^{\prime}+4 \mu_{A^{4}}\right\}+\cdots .
$$

Perhaps the most interesting application of these formulas is to the proton. If we take Eqs. (3.23) and (3.24) literally, we must in that case include in $\sigma_{T}$ the cross section for electron pair production in the Coulomb field of the proton as well as the cross section for such processes as photomeson production. However, at energies above a few Mev, but still small compared to the meson rest energy, the Delbrück scattering arising from real and virtual pair production has an angular distribution sharply peaked in the forward direction; on the other hand the Thomson and Rayleigh scattering and the scattering from the magnetic moment should have rather slowly varying angular distributions at these energies. We may therefore omit the Delbrück scattering, but leave Eq. (3.24) otherwise unchanged, if we evaluate $d \sigma / d \Omega$ at an angle near $0^{\circ}$ but far enough away so that the Delbrück scattering is small. In that case the principal contribution to $\sigma_{T}$ is the cross section $\sigma_{P}$ for the photopion effect. Empirical data on this process indicate the relation ${ }^{12}$

$$
\frac{1}{2 \pi^{2}} \int_{m_{\pi}}^{\infty} \frac{\sigma_{P}\left(q^{\prime}\right)}{q^{\prime 2}} d q^{\prime} \simeq \frac{e^{2}}{3 m_{\pi}{ }^{2} M}
$$

${ }_{12}$ Gell-Mann, Goldberger, and Thirring, Phys. Rev. 95, 1612 (1954). 
Thus we find

$\left(\frac{d \sigma}{d \Omega}\right)_{\text {near } 0^{\circ}} \simeq \frac{e^{4}}{M^{2}}+\frac{2 q^{2} e^{4}}{3 M^{2}} \frac{1}{m_{\pi}^{2}}\left\{-1+\frac{3 m_{\pi}^{2} \beta_{A}^{4}}{8 M^{2}}\right\}+\cdots$,

where $\beta_{A}$ is the anaomalous magnetic moment in nuclear Bohr magnetons. Since $3 m_{\pi}^{2} \beta_{A}^{4} / 8 M^{2} \simeq 0.08$, we see that near the forward direction the scattering by the magnetic moment is greatly overcompensated by the interference of Thomson and Rayleigh scattering. We cannot say with certainty, of course, that this situation persists at most angles, but it seems likely.

So far we have treated the scatterer as a "particle," but it seems reasonable that Eq. (3.2) should be valid for any system of $\operatorname{spin} \frac{1}{2}$, whether elementary or complex, and that the expansion in frequency should be possible when the photon energy is small compared to the energy of the first excited state of the system. Thus complex nuclei of spin $\frac{1}{2}$ may be treated, as well as the proton.

The generalization of the result to other spins is an interesting problem. For spin zero, the term in the scattering amplitude linear in frequency must vanish in order that the symmetry condition (3.5) be satisfied. For spins higher than $\frac{1}{2}$, we conjecture that it is still possible to express the linear term in terms of static properties of the system.

We acknowledge with thanks conversations with Professors G. Wentzel and V. L. Telegdi concerning classical methods of calculation.

\title{
Calculation of Potentials from the New Tamm-Dancoff Equation
}

\author{
J. C. TAYLOR \\ Peterhouse, Cambridge, England
}

(Received July 26, 1954)

\begin{abstract}
This note is about the construction of energy-independent potentials from the Tamm-Dancoff equations, by a systematic method of Klein's. It is shown that, when the method is applied to the new Tamm-Dancoff equation of Dyson, the difficulty of the spurious singularities can be overcome. It is proved further that the old and new Tamm-Dancoff methods give the same potential in the adiabatic limit.
\end{abstract}

\section{INTRODUCTION}

$I^{1}$ $\mathrm{N}$ this note, we are concerned with the use of the Tamm-Dancoff methods ${ }^{1}$ to derive, for the twonucleon system, a single integral equation like that used, for instance, by Lévy. ${ }^{2}$ The old Tamm-Dancoff method (O.T.D.), which has been used in the past, suffers from infinite vacuum-to-vacuum terms. These do not occur in the new Tamm-Dancoff method (N.T.D.) of Dyson. ${ }^{3}$ N.T.D. has also the advantage of being generally closer than O.T.D. to the covariant, renormalizable theories.

The kernel of the N.T.D. integral equation contains "spurious" poles, ${ }^{4}$ which are not at the threshold for any real physical process. We thus have the problem of imposing boundary conditions on the amplitude which satisfies the integral equation, so that it should have no singularities arising from the spurious poles. This problem, which amounts to the definition of the vacuum state in the Dyson amplitudes, has not yet been solved.

The purpose of this note is to examine what form the problem of the spurious singularities takes when the kernel of the N.T.D. integral equation is considered as a generalized potential. The kernel is dependent upon the

${ }^{1}$ S. M. Dancoff, Phys. Rev. 78, 382 (1950).

2 M. M. Lévy, Phys. Rev. 88, 72 (1952).

3 F. J. Dyson, Phys. Rev. 90, 994 (1953).

4 F. J. Dyson, Phys. Rev. 91, 1543 (1953). total energy, and so does not give a straightforward eigenvalue equation for bound states. We adopt a systematic method of eliminating the energy dependence, which has been proposed by Klein. ${ }^{5}$ Klein's actual suggestion was for the adiabatic limit; but in Sec. 1 we give a natural generalization of his method, which, while it would probably be useful only near the adiabatic limit, enables one to obtain some general properties of the energy-independent potential.

In Sec. 1, we also give a method of generating energyindependent potentials, using the four-dimensional formalism of N.T.D. of Mathews and Salam. ${ }^{6}$ This method, which agrees with Klein's except for a few special terms, is the basis of our general results.

In Sec. 2, we show that, for the energy-independent potential, the problem of the spurious singularities reduces to the simpler question of how to define the poles in a certain integral. It is no longer a boundary condition problem. The essential step in this demonstration is a generalization of a result already given by Klein ${ }^{7}$ for the fourth order. This states that, if the total energy is replaced by the kinetic energy, the kernel is free of spurious poles on a suitable rearrangement of terms.

${ }^{5}$ A. Klein, Phys. Rev. 94, 195 (1954).

${ }^{6}$ Quoted by the present author in reference 9.

7 A. Klein, Phys. Rev. 95, 1653 (1954). 